\title{
Linear Regression for Astronomical Data with Measurement Errors and Intrinsic Scatter
}

\author{
Michael G. Akritas \\ Department of Statistics, The Pennsylvania State University, State College PA 16802 \\ mga@stat.psu.edu \\ and \\ Matthew A. Bershady ${ }^{1}$ \\ Department of Astronomy \& Astrophysics, The Pennsylvania State University, State \\ College PA 16802 \\ mab@astro.psu.edu
}

To appear in the Astrophysical Journal, October 10, 1996 issue, Vol. 470

Received —

\footnotetext{
${ }^{1}$ Hubble Fellow
} 


\begin{abstract}
Two new methods are proposed for linear regression analysis for data with measurement errors. Both methods are designed to accommodate intrinsic scatter in addition to measurement errors. The first method is a direct extension of the ordinary least squares (OLS) estimator to allow for measurement errors. It is quite general in that a) it allows for measurement errors on both variables, b) it allows the measurement errors for the two variables to be dependent, c) it allows the magnitudes of the measurement errors to depend on the measurements, and d) other 'symmetric' lines such as the bisector and the orthogonal regression can be constructed. We refer to this method as BCES estimators (for Bivariate Correlated Errors and intrinsic Scatter). The second method is a weighted least squares (WLS) estimator, which applies only in the case where the 'independent' variable is measured without error and the magnitudes of the measurement errors on the 'dependent' variable are independent from the measurements.

Several applications are made to extragalactic astronomy: The BCES method, when applied to data describing the color-luminosity relations for field galaxies, yields significantly different slopes than OLS and other estimators used in the literature. Simulations with artificial data sets are used to evaluate the small sample performance of the estimators. Unsurprisingly, the least-biased results are obtained when color is treated as the dependent variable. The Tully-Fisher relation is another example where the BCES method should be used because errors in luminosity and velocity are correlated due to inclination corrections. We also find, via simulations, that the WLS method is by far the best method for the Tolman surface-brightness test, producing the smallest variance in slope by an order of magnitude. Moreover, with WLS it is not necessary to "reduce" galaxies to a fiducial surface-brightness, since this model incorporates intrinsic scatter.
\end{abstract}

Subject headings: statistical methods: analytical, numerical - galaxies, cosmology: color-luminosity relation, Tully-Fisher relation, Tolman test 


\section{Introduction}

Linear regression analysis is used extensively in everyday astronomical research. The distinguishing feature of many astronomical data sets is the presence of intrinsic scatter in addition to heteroscedastic measurement errors (i.e. the size of the error can vary from observation to observation). A few notable examples in extra-galactic astronomy include relations between X-ray temperatures and velocity dispersions for galaxy clusters, the color-luminosity relations for field galaxies, the Tully-Fisher relation (and other "Fundamental Plane" relations), and the Tolman test. In this paper we consider the latter three examples. Neglect of measurement errors and intrinsic can bias the derived slopes of these relations, thus potentially leading to incorrect astrophysical deductions. Willick (1991) has performed a detailed study of the effects of intrinsic scatter and measurement error on the estimated slope of the Tully-Fisher relation. An additional issue pertains to biases associated with the correlation of measurement errors between observables. This occurs, for example, in color-luminosity relations and the Tully-Fisher relation. There are some cases where the measurement error is negligible in one variable. For example, in the case of the Tolman surface-brightness test, the error in redshift is usually negligible compared to the error and intrinsic scatter in surface-brightness.

Regression model found in statistics text books rarely accommodate heteroscedastic measurement errors in more than one variable, and the recent paper Isobe et al. (1991) (IFAB hereafter) deals exclusively with data that have no measurement errors. Indeed, accommodating heteroscedastic measurement errors and intrinsic scatter is mentioned in Feigelson \& Babu (1992) as one of the outstanding problems in linear regression.

The only currently available regression methods that deal with heteroscedastic measurement errors are based on the assumption that the true variables (in the absence of measurement error) have no intrinsic scatter. That is, the true points are assumed to lie exactly on a straight line, which implies they have correlation one. Software packages which perform regressions under this assumption are mentioned in Feigelson \& Babu (1992), including ORDPACK (Boggs et al. 1990), which also does nonlinear regression. A more accessible reference is Press et al. (1988). This assumption, however, is violated in many astronomical data sets.

In this paper we address the important problem of fitting regression models with data having heteroscedastic measurement errors of known standard deviation, and entirely unknown intrinsic scatter. We define a statistical model for data with astronomical (heteroscedastic) measurement errors which allows the possibility of correlated errors between both variables of interest, and the possibility that the size of the measurement 
error depends on the observation. This model should prove useful for addressing other problems with such data, including intrinsic variance function estimation, goodness-of-fit, comparing $k$ multivariate samples etc). Very important is the distinction we draw between the case where the size of the measurement error (standard deviation in statistical parlance) depends on the measurement and the case where it does not. Both cases are equally common in astronomical data sets (e.g. background-limited versus source-limited observations). However, procedures that weigh the measurements according to the variance of the measurement error can give biased results if this variance depends on the observation, as we discuss in the next section.

We describe here two different regression methods. Both of our methods pertain only to linear (as opposed to nonlinear) regression and are based on transparent ideas that make them very intuitive. The first method is a direct generalization of the OLS estimator which applies quite generally. The second method is a weighted least squares (WLS) estimator which applies when only the 'response' variable is subject to measurement error and the size of the measurement error does not depend on the observation.

We only consider simple linear regression here (i.e. only one 'explanatory' variable); extensions of this method to multiple regressions will appear in a sequel paper. The paper is organized as follows. In the next section we introduce the basic idea of our method. The statistical model for data with measurement errors is presented in subsection 2.1. In subsection 2.1 we consider the general case where both the response and the explanatory variable are subject to potentially correlated measurement errors, and the magnitude of these errors may depend on the measurements. We use the acronym $\operatorname{BCES}\left(X_{2} \mid X_{1}\right)$ to denote the present generalization of the $\operatorname{OLS}\left(X_{2} \mid X_{1}\right)$, which minimizes the residuals in $X_{2}$ (i.e. $X_{2}$ is the dependent variable). In subsection 2.2 we consider the case where only the response variable is subject to measurement error whose magnitude does not depend on the measurement, and we introduce a competing procedure based on WLS. In Section 3 we study other versions of the first method, namely the BCES-bisector and BCES-orthogonal regression; these regression lines are defined in terms of $\operatorname{BCES}\left(X_{2} \mid X_{1}\right)$ and $\operatorname{BCES}\left(X_{1} \mid X_{2}\right)$. In Section 4 we apply these methods to an astronomical data set and use simulations as a methodological tool to investigate the small-sample performance of the four BCES estimators (for color-luminosity relations) and the WLS estimator (for the Tolman test). Relevance to the Tully-Fisher relation is discussed. We consider more general application of BCES in Section 5. The mathematical derivations are given in the Appendix. 


\section{Simple Regression}

As explained in the introduction, observed astrophysical correlations exhibit are expected to exhibit scatter due to both intrinsic and measurement error. These two sources of scatter must be recognized and separately treated to avoid biases. However, different kinds of measurement errors have different effects - some being benign. There are two basic distinctions: a) whether or not the measurement error is in the independent variable, and b) whether or not the magnitude of the measurement error depends on the measurement.

It is well documented in the statistical literature that the OLS slope is biased if there is measurement error in the independent variable. Measurement errors only in the dependent variable are less critical: Provided the measurement error is independent from the observation, OLS is valid but not efficient. The technical term "not efficient" means that there exists another estimator which has smaller scatter (and therefore results in narrower confidence intervals). In particular, if there is no measurement error in the independent variable and the measurement error in the dependent variable is independent from the measurement, the WLS estimator in subsection 2.3 is more efficient than the OLS estimator. On the other hand, if the size of the measurement error depends on the measurement, neither the OLS estimator nor the WLS estimator of subsection 2.3 are valid, even if the independent variable is measured without error, and even if there is no intrinsic scatter. The technical term "not valid" means that the estimator is biased even for large samples; as a consequence, the larger the sample size, the less likely it will be for a confidence interval based on it to contain the true value. The bias can get worse if there is measurement errors in both variables, and more so if the measurement errors are correlated.

In subsection 2.2 we present an extension of the OLS slope which is valid in all cases. This is also the "conservative" estimator that should be used when one is unclear about the

prevailing conditions. When the independent variable is measured without error and one is certain that the magnitude of the measurement error does not depend on the observation, the WLS estimator of subsection 2.3 is more efficient and therefore recommended. First, however, we need to set the notation and formulate a statistical model for data with heteroscedastic measurement errors. This is done in subsection 2.1. Inadvertently, these sections are technical. 


\subsection{A Statistical Model}

Let the variables of interest be denoted by $\left(X_{1 i}, X_{2 i}\right)$ and the observed data be denoted by

$$
\left(Y_{1 i}, Y_{2 i}, \mathbf{V}_{i}\right), i=1, \ldots n
$$

where for each $i, \mathbf{V}_{i}$ is a symmetric $2 \times 2$ matrix with elements denoted by $V_{11, i}, V_{22, i}$, and $V_{12, i}$, for the two diagonal and the common off diagonal elements, respectively. The observed data are related to the unobserved variables of interest by

$$
Y_{1 i}=X_{1 i}+\epsilon_{1 i}, \text { and } Y_{2 i}=X_{2 i}+\epsilon_{2 i},
$$

where the errors $\left(\epsilon_{1 i}, \epsilon_{2 i}\right)$ have a joint bivariate distribution with zero mean and covariance matrix $\mathbf{V}_{i}$, for all $i$. In this model we allow $\mathbf{V}_{i}$ to depend on $\left(Y_{1 i}, Y_{2 i}\right)$ and thus, implicitly on $\left(X_{1 i}, X_{2 i}\right)$. Thus we do not require that $\left(\epsilon_{1 i}, \epsilon_{2 i}\right)$ be independent from $\left(X_{1 i}, X_{2 i}\right)$. However, we assume that $\mathbf{V}_{i}$ is the only aspect of the distribution of $\left(\epsilon_{1 i}, \epsilon_{2 i}\right)$ that depends on $\left(Y_{1 i}, Y_{2 i}\right)$. In other words, we assume that, given $\mathbf{V}_{i},\left(\epsilon_{1 i}, \epsilon_{2 i}\right)$ is independent from $\left(X_{1 i}, X_{2 i}\right)$.

The intuitive meaning of the technical assumption that "given $\mathbf{V}_{i},\left(\epsilon_{1 i}, \epsilon_{2 i}\right)$ is independent from $\left(X_{1 i}, X_{2 i}\right)$ " is that $\epsilon_{1 i}$, for example, is equally likely to be positive or negative for any value of $X_{1 i}$, and the size of its absolute value is governed (in addition to the type of the measurement error distribution) by the magnitude of $V_{11, i}$ which is given. All astronomical data sets that we are aware of comply to this assumption.

In most cases, the measurement errors for the two variables are independent (so $V_{12, i}=0$ for all $i$ ), and the observed data is of the form 1

$$
\left(Y_{1 i}, Y_{2 i}, V_{11, i}, V_{22, i}\right) \text {, }
$$

with $V_{k k, i}$ denoting the variance of $\epsilon_{k i}, k=1,2$.

It is assumed that the variables of interest follow the usual simple regression model

$$
X_{2 i}=\alpha_{1}+\beta_{1} X_{1 i}+e_{i},
$$

\footnotetext{
${ }^{2}$ Very often, astronomical data sets will not give explicitly the magnitude of the uncertainty of the errors (i.e. $V_{11, i}, V_{22, i}$ ). Instead the uncertainty is reported in the form of $(1-\alpha) 100 \%$ (e.g. 95\%) confidence intervals $Y_{1 i} \pm c_{1 i}, Y_{2 i} \pm c_{2 i}$. In this case the $V$ 's can be recovered from the relation $c_{k i}=z_{\alpha / 2} \sqrt{V_{k k, i}}$, for $k=1,2$, where $z_{\alpha / 2}$ is the $(1-\alpha / 2) 100$-th percentile of the standard normal distribution.
} 
where $e_{i}$ is assumed to have zero mean and finite variance. The terminology "intrinsic scatter" (or "intrinsic dispersion") is commonly used to indicate the variance or standard deviation of $e_{i}$. We want to estimate the regression coefficients $\alpha_{1}, \beta_{1}$ and also estimate the uncertainties of these estimators using the data in (11).

\subsection{The $\operatorname{BCES}\left(X_{2} \mid X_{1}\right)$ Estimator}

The estimator $\operatorname{BCES}\left(X_{2} \mid X_{1}\right)$ (see the Abstract and Introduction for explanation of the acronym) to be proposed here is an extension of the $\operatorname{OLS}\left(X_{2} \mid X_{1}\right)$ estimator which is valid for all data sets that fit the measurement error model specified in subsection 2.1. This estimator is based on the fact that the parameters of (3) are related to the moments of the bivariate distribution of $\left(X_{1 i}, X_{2 i}\right)$. In particular,

$$
\beta_{1}=\frac{C\left(X_{1 i}, X_{2 i}\right)}{V\left(X_{1 i}\right)} \text {, and } \alpha_{1}=E\left(X_{2 i}\right)-\beta_{1} E\left(X_{1 i}\right),
$$

where $C\left(X_{1 i}, X_{2 i}\right)$ denotes the covariance of $X_{1 i}$ and $X_{2 i}, V\left(X_{1 i}\right)$ denotes the variance of $X_{1 i}$ and $E$ denotes expected value. In the case of no measurement errors, the OLS estimators are simply moment estimators, so they are obtained by replacing the population moments in (四) by sample moments. The proposed estimators generalize the OLS estimators by replacing the population moments in (4) by moment estimators obtained from the observed data (1). These moment estimators are based on the following results: For $k=1$ or 2 we have

$$
\begin{aligned}
E\left(Y_{k i}\right) & =E\left(X_{k i}\right) \\
V\left(Y_{k i}\right) & =V\left(X_{k i}\right)+E\left(V_{k k, i}\right) \\
C\left(Y_{1 i}, Y_{2 i}\right) & =C\left(X_{1 i}, X_{2 i}\right)+E\left(V_{12, i}\right) .
\end{aligned}
$$

The proof of these results is given in the Appendix.

Using relations (5), (6), (7) and relation (4) we can express the regression parameters $\alpha_{1}, \beta_{1}$ in terms of the population moments of the observed data. Thus,

$$
\beta_{1}=\frac{C\left(Y_{1 i}, Y_{2 i}\right)-E\left(V_{12, i}\right)}{V\left(Y_{1 i}\right)-E\left(V_{11, i}\right)} \text {, and } \alpha_{1}=E\left(Y_{2 i}\right)-\beta E\left(Y_{1 i}\right) .
$$

This relation suggests the following extension of the OLS estimator to data with measurement errors,

$$
\hat{\beta}_{1}=\frac{\sum_{i=1}^{n}\left(Y_{1 i}-\bar{Y}_{1}\right)\left(Y_{2 i}-\bar{Y}_{2}\right)-\sum_{i=1}^{n} V_{12, i}}{\sum_{i=1}^{n}\left(Y_{1 i}-\bar{Y}_{1}\right)^{2}-\sum_{i=1}^{n} V_{11, i}}
$$




$$
\hat{\alpha}_{1}=\bar{Y}_{2}-\hat{\beta}_{1} \bar{Y}_{1}
$$

These are the $\operatorname{BCES}\left(X_{2} \mid X_{1}\right)$ estimators of the slope and intercept which generalize the $\operatorname{OLS}\left(X_{2} \mid X_{1}\right)$ estimators. The way the $\operatorname{BCES}\left(X_{2} \mid X_{1}\right)$ slope estimator adjusts for the presence of measurement errors is quite obvious from (9). Namely, if the errors are correlated, the observed covariance between $Y_{1}$ and $Y_{2}$ is biased. Therefore, the numerator of $\hat{\beta}_{1}$ consists of a "debiased" sample covariance. Similarly, the observed variance of $Y_{1}$ is biased necessitating the "debiased" sample variance seen in the denominator of $\hat{\beta}_{1}$.

It will be shown in the Appendix that these estimators all have, asymptotically, a zero mean normal distribution. To give expressions for their variances, we need the following notation. Let

$$
\begin{aligned}
\xi_{1 i} & =\frac{\left(Y_{1 i}-E\left(Y_{1 i}\right)\right)\left(Y_{2 i}-\beta_{1} Y_{1 i}-\alpha_{1}\right)+\beta_{1} V_{11, i}-V_{12, i}}{V\left(Y_{1 i}\right)-E\left(V_{11, i}\right)} \\
\zeta_{1 i} & =Y_{2 i}-\beta_{1} Y_{1 i}-E\left(Y_{1 i}\right) \xi_{1 i},
\end{aligned}
$$

and let $\hat{\xi}_{1 i}, \hat{\zeta}_{1 i}$ be obtained by substituting the unknown quantities in $\xi_{i 1}, \zeta_{1 i}$ by their obvious estimators (i.e. substitute sample means in place of population means, sample variances in place of population variances, and $\hat{\beta}_{1}, \hat{\alpha}_{1}$ in place of $\left.\beta_{1}, \alpha_{1}\right)$. Finally, let $\overline{\hat{\xi}}_{1}\left(\overline{\hat{\zeta}}_{1}\right)$ denote the arithmetic average of the $\hat{\xi}_{1 i}\left(\hat{\zeta}_{1 i}\right)$ and set

$$
\begin{aligned}
& \hat{\sigma}_{\beta_{1}}^{2}=n^{-1} \sum_{i=1}^{n}\left(\hat{\xi}_{1 i}-\overline{\hat{\xi}}_{1}\right)^{2} \\
& \hat{\sigma}_{\alpha_{1}}^{2}=n^{-1} \sum_{i=1}^{n}\left(\hat{\zeta}_{1 i}-\overline{\hat{\zeta}}_{1}\right)^{2} .
\end{aligned}
$$

Then the variance of $\hat{\beta}_{1}$ is estimated by $\widehat{V}\left(\hat{\beta}_{1}\right)=n^{-1} \hat{\sigma}_{\beta_{1}}^{2}$; thus, the asymptotic normality shown in the Appendix implies that a $(1-\alpha) 100 \%$ confidence interval for $\beta_{1}$ is

$$
\hat{\beta}_{1} \pm z_{\alpha / 2} \hat{\sigma}_{\beta_{1}} n^{-1 / 2} \text {. }
$$

Similarly the variance of $\hat{\alpha}_{1}$ is estimated by $\widehat{V}\left(\hat{\alpha}_{1}\right)=n^{-1} \hat{\sigma}_{\alpha_{1}}^{2}$ with a similar confidence interval implied from the asymptotic normality.

Finally let $\hat{\sigma}_{\beta_{1}, \alpha_{1}}$ be the sample covariance obtained from $\left(\hat{\xi}_{1 i}, \hat{\zeta}_{1 i}\right)$. Then the covariance between $\hat{\beta}_{1}$ and $\hat{\alpha}_{1}$ is estimated by

$$
\widehat{\operatorname{Cov}}\left(\hat{\beta}_{1}, \hat{\alpha}_{1}\right)=n^{-1} \hat{\sigma}_{\beta_{1}, \alpha_{1}} .
$$

This estimated covariance function can be used for constructing a simultaneous confidence ellipsoid for $\hat{\beta}_{1}$ and $\hat{\alpha}_{1}$. See for example Johnson \& Wichern (1988). 


\subsection{Only the Response Variable with Measurement Error}

In this subsection we describe a WLS estimator for the case that $X_{1 i}$ is observed without error. This estimator requires the additional assumption that the measurement error in $X_{2 i}$ is independent of $X_{2 i}$. The main idea behind this estimator is that, in the presence of nonnegligible intrinsic scatter, the optimal weight for each observation is made up both from the variance of the corresponding measurement error and the intrinsic scatter. Thus, to determine the optimal weight we first need to estimate the intrinsic scatter. We proceed with a formal description of this method.

In the case that $V_{11, i}=0$ for all $i$ (so also $V_{12, i}=0$ ), relations (2) and (3) imply

$$
\begin{aligned}
Y_{2 i} & =X_{2 i}+\epsilon_{2 i} \\
& =\alpha_{1}+\beta_{1} X_{1 i}+e_{i}+\epsilon_{2 i} \\
& =\alpha_{1}+\beta_{1} X_{1 i}+e_{i}^{*},
\end{aligned}
$$

where we have set $e_{i}^{*}=e_{i}+\epsilon_{2 i}$. This is the typical setting for the application of WLS, provided that the variance of $e_{i}^{*}$ is independent of $Y_{2 i}$. To do so, however, we need to estimate the variance of $e_{i}^{*}$. Note that, under the assumption made,

$$
V\left(e_{i}^{*}\right)=V\left(e_{i}\right)+V_{22, i} .
$$

Thus $V\left(e_{i}^{*}\right)$ is unknown because the intrinsic scatter $V\left(e_{i}\right)$ is unknown. We propose the following method for estimating $V\left(e_{i}\right)$.

Step 1. Obtain $\hat{\alpha}_{O L S}, \hat{\beta}_{O L S}$ by a direct application of OLS to the data $\left(Y_{2 i}, X_{1 i}\right)$.

Step 2. Calculate the residuals

$$
R_{i}=Y_{2 i}-\hat{\alpha}_{O L S}-\hat{\beta}_{O L S} X_{1 i}
$$

Step 3. Obtain the estimator of $V\left(e_{i}\right)$ from

$$
\widehat{V\left(e_{i}\right)}=n^{-1} \sum_{i=1}^{n}\left(R_{i}-\bar{R}\right)^{2}-n^{-1} \sum_{i=1}^{n} V_{22, i}
$$

It can be shown that the estimator of $V\left(e_{i}\right)$ described in (16) is consistent. Next, set

$$
\widehat{V\left(e_{i}^{*}\right)}=\hat{\sigma}_{i}^{* 2}=\widehat{V\left(e_{i}\right)}+V_{22, i}
$$


and let $A$ be the $n \times n$ matrix with diagonal elements $\hat{\sigma}_{i}^{* 2}$ and with all off-diagonal elements equal to zero. In terms of $A$, a general formula for the WLS estimator is given in Arnold (1981). For the present simple regression problem, this formula gives the following WLS estimators for $\beta_{1}$,

$$
\begin{aligned}
\hat{\beta}_{W L S} & =\frac{\sum \hat{\sigma}_{i}^{*-2} \sum \hat{\sigma}_{i}^{*-2} X_{1 i} Y_{2 i}-\sum \hat{\sigma}_{i}^{*-2} X_{1 i} \sum \hat{\sigma}_{i}^{*-2} Y_{2 i}}{\sum \hat{\sigma}_{i}^{*-2} \sum \hat{\sigma}_{i}^{*-2} X_{1 i}^{2}-\left(\sum \hat{\sigma}_{i}^{*-2} X_{1 i}\right)^{2}} \\
\hat{\alpha}_{W L S} & =\frac{\sum \hat{\sigma}_{i}^{*-2} X_{1 i}^{2} \sum \hat{\sigma}_{i}^{*-2} Y_{2 i}-\sum \hat{\sigma}_{i}^{*-2} X_{1 i} \sum \hat{\sigma}_{i}^{*-2} X_{1 i} Y_{2 i}}{\sum \hat{\sigma}_{i}^{*-2} \sum \hat{\sigma}_{i}^{*-2} X_{1 i}^{2}-\left(\sum \hat{\sigma}_{i}^{*-2} X_{1 i}\right)^{2}} .
\end{aligned}
$$

Variance estimates for the WLS estimators are

$$
\begin{aligned}
\widehat{V}\left(\hat{\beta}_{W L S}\right) & =\frac{\sum \hat{\sigma}_{i}^{*-2}}{\sum \hat{\sigma}_{i}^{*-2} \sum \hat{\sigma}_{i}^{*-2} X_{1 i}^{2}-\left(\sum \hat{\sigma}_{i}^{*-2} X_{1 i}\right)^{2}} \\
\widehat{V}\left(\hat{\alpha}_{W L S}\right) & =\frac{\sum \hat{\sigma}_{i}^{*-2} X_{1 i}^{2}}{\sum \hat{\sigma}_{i}^{*-2} \sum \hat{\sigma}_{i}^{*-2} X_{1 i}^{2}-\left(\sum \hat{\sigma}_{i}^{*-2} X_{1 i}\right)^{2}} .
\end{aligned}
$$

Note that these are conditional (given $X_{11}, \ldots, X_{1 n}$ ) estimates of the variance of the WLS estimators and, when the $\hat{\sigma}_{i}^{*}$ are all equal, reduce to the usual variance estimates of the OLS estimator (Draper \& Smith, 1981).

\section{Other Estimators}

Text books in Statistics rarely mention a slope estimator other than the $\operatorname{OLS}\left(X_{2} \mid X_{1}\right)$. However, for some data sets in astronomy (and other sciences) it is not clear which variable should be treated as the independent and which as the dependent. Since OLS $\left(X_{1} \mid X_{2}\right)$ gives a different slope, astronomers have invented the bisector slope. This corresponds to the line that bisects the $\operatorname{OLS}\left(X_{2} \mid X_{1}\right)$ and $\operatorname{OLS}\left(X_{1} \mid X_{2}\right)$ lines. In addition, astronomers also use orthogonal least squares (which finds the line that minimizes the squared orthogonal distances) and some others. Astronomers are well aware that, for any given data set, each of the above slopes will probably be different. However, the concept that theses sample slopes estimate different population slopes is more elusive. In other words, the true $\operatorname{OLS}\left(X_{2} \mid X_{1}\right)$ slope is different from the true $\operatorname{OLS}\left(X_{1} \mid X_{2}\right)$ slope as well as from all other true slopes. In still different words, the $\operatorname{OLS}\left(X_{2} \mid X_{1}\right)$ slope obtained from any given data set is a biased estimator of the true $\operatorname{OLS}\left(X_{1} \mid X_{2}\right)$ slope and all other true slopes and, for large enough sample sizes, confidence intervals around each of the sample slopes will not overlap. This implies, of course, that there is no such thing as "true slope", but there is a true OLS $\left(X_{1} \mid X_{2}\right)$ 
slope, a true bisector slope etc. This point is raised in IFAB, but it is critical to explain again here: Statistics cannot offer guidance as to which of the true slopes is appropriate for any given situation. This decision is up to the scientist. Statistics offers variance formulas that indicate how accurately each sample slope is estimating the corresponding true slope. However, since these formulas are asymptotic (meaning valid for large samples) simulation studies are recommended to examine the small sample performance of each estimator.

In this section we present such slopes and intercepts for data sets with measurement errors. Let $\hat{\beta}_{2}, \hat{\beta}_{3}$, and $\hat{\beta}_{4}$ denote the $\operatorname{OLS}\left(X_{1} \mid X_{2}\right)$, bisector, and orthogonal regression slope, respectively. The corresponding intercepts are

$$
\hat{\alpha}_{\iota}=\bar{Y}_{2}-\hat{\beta}_{\iota} \bar{Y}_{1}, \quad \iota=2,3,4 .
$$

The formula for the $\operatorname{OLS}\left(X_{1} \mid X_{2}\right)$ slope is

$$
\hat{\beta}_{2}=\frac{\sum_{i=1}^{n}\left(Y_{2 i}-\bar{Y}_{2}\right)^{2}-\sum_{i=1}^{n} \Sigma_{22, i}}{\sum_{i=1}^{n}\left(Y_{1 i}-\bar{Y}_{1}\right)\left(Y_{2 i}-\bar{Y}_{2}\right)-\sum_{i=1}^{n} \Sigma_{12, i}} .
$$

The slopes $\hat{\beta}_{3}$, and $\hat{\beta}_{4}$ are given in terms of the slopes of $\hat{\beta}_{1}$, and $\hat{\beta}_{2}$ according to the formulas given in Table 1 of IFAB. The asymptotic normality of all slope and intercept estimators follows by arguments similar to those in Appendix A of IFAB and the proof in the present Appendix. To estimate the variance of each estimator, consider the notation. Let

$$
\begin{aligned}
& \hat{\xi}_{2 i}=\frac{\left(Y_{2 i}-\bar{Y}_{2}\right)\left(Y_{2 i}-\hat{\beta}_{2} Y_{1 i}-\hat{\alpha}_{2}\right)+\hat{\beta}_{2} V_{12, i}-V_{22, i}}{S_{Y_{1}, Y_{2}}-\bar{V}_{12}} \\
& \hat{\zeta}_{2 i}=Y_{2 i}-\hat{\beta}_{2} Y_{1 i}-\bar{Y}_{1} \hat{\xi}_{2 i} \\
& \hat{\xi}_{3 i}=\frac{\left(1+\hat{\beta}_{2}^{2}\right) \hat{\beta}_{3}}{\left(\hat{\beta}_{1}+\hat{\beta}_{2}\right) \sqrt{\left(1+\hat{\beta}_{1}^{2}\right)\left(1+\hat{\beta}_{2}^{2}\right)}} \hat{\xi}_{1 i}+\frac{\left(1+\hat{\beta}_{1}^{2}\right) \hat{\beta}_{3}}{\left(\hat{\beta}_{1}+\hat{\beta}_{2}\right) \sqrt{\left(1+\hat{\beta}_{1}^{2}\right)\left(1+\hat{\beta}_{2}^{2}\right)}} \hat{\xi}_{2 i}, \\
& \hat{\zeta}_{3 i}=Y_{2 i}-\hat{\beta}_{3} Y_{1 i}-E\left(Y_{1 i}\right) \hat{\xi}_{3 i}, \\
& \hat{\xi}_{4 i}=\frac{\hat{\beta}_{4}}{\hat{\beta}_{1}^{2} \sqrt{4+\left(\hat{\beta}_{2}-\hat{\beta}_{1}^{-1}\right)^{2}}} \hat{\xi}_{1 i}+\frac{\hat{\beta}_{4}}{\sqrt{4+\left(\hat{\beta}_{2}-\hat{\beta}_{1}^{-1}\right)^{2}}} \hat{\xi}_{2 i}, \\
& \hat{\zeta}_{4 i}=Y_{2 i}-\hat{\beta}_{4} Y_{1 i}-E\left(Y_{1 i}\right) \hat{\xi}_{4 i},
\end{aligned}
$$

where $S_{Y_{1}, Y_{2}}=n^{-1} \sum_{i=1}^{n}\left(Y_{1 i}-\bar{Y}_{1}\right)\left(Y_{2 i}-\bar{Y}_{2}\right)$, and $\bar{Y}_{1}, \bar{Y}_{2}, \bar{V}_{12}$ are the arithmetic averages of $Y_{1 i}, Y_{2 i}, V_{22, i}$, respectively. Also, for $\iota=2,3,4$, let $\hat{\sigma}_{\beta_{\iota}}^{2}, \hat{\sigma}_{\alpha_{\iota}}^{2}$, be the sample variances obtained from $\hat{\xi}_{\iota i}, i=1, \ldots, n$ and $\hat{\zeta}_{\iota i}, i=1, \ldots, n$, respectively. Arguing as in the Appendix, the variances of $\hat{\beta}_{\iota}$ and $\hat{\alpha}_{\iota}$ are estimated by

$$
\widehat{V}\left(\hat{\beta}_{\iota}\right)=n^{-1} \hat{\sigma}_{\beta_{\iota}}^{2}, \quad \widehat{V}\left(\hat{\alpha}_{\iota}\right)=n^{-1} \hat{\sigma}_{\alpha_{\iota}}^{2}
$$


respectively, $\iota=2,3,4$. In addition, the covariance between $\hat{\beta}_{\iota}$ and $\hat{\alpha}_{\iota}$ is estimated by

$$
\widehat{\operatorname{Cov}}\left(\hat{\beta}_{\iota}, \hat{\alpha}_{\iota}\right)=n^{-1} \hat{\sigma}_{\beta_{\iota}, \alpha_{\iota}}
$$

where $\hat{\sigma}_{\beta_{\iota}, \alpha_{\iota}}$ is the sample covariance obtained from $\left(\hat{\xi}_{\iota i}, \hat{\zeta}_{\iota i}\right), \iota=2,3,4$.

We note in closing that the variance of $\hat{\beta}_{3}$ is related to the variances of $\hat{\beta}_{1}$ and $\hat{\beta}_{2}$ through the formula given in Table 1 of IFAB. In particular,

$$
\begin{gathered}
\hat{V}\left(\hat{\beta}_{3}\right)=\frac{\hat{\beta}_{3}^{2}}{\left(\hat{\beta}_{1}+\hat{\beta}_{2}\right)^{2}\left(1+\hat{\beta}_{1}^{2}\right)\left(1+\hat{\beta}_{2}^{2}\right)}\left[\left(1+\hat{\beta}_{2}^{2}\right)^{2} \widehat{V}\left(\hat{\beta}_{1}\right)+\right. \\
+\left(1+\hat{\beta}_{1}^{2}\right)\left(1+\hat{\beta}_{2}^{2}\right) \widehat{\operatorname{Cov}}\left(\hat{\beta}_{1}, \hat{\beta}_{2}\right) \\
\left.+\left(1+\hat{\beta}_{1}^{2}\right)^{2} \widehat{V}\left(\hat{\beta}_{2}\right)\right] .
\end{gathered}
$$

It can be shown analytically, that the value obtained from the formula in (32) will always be somewhat larger than that obtained from (30) with $\iota=3$ but this difference will be negligible for large sample sizes. A similar remark applies for the variance of $\hat{\beta}_{4}$.

Some simulation studies examining the small sample performance of these estimators and their estimated variances are presented in the next section in reference to a particular application.

\section{Example Applications To Real Data}

\subsection{Correlated errors and intrinsic scatter in $X_{1}$ and $X_{2}$}

\subsubsection{Color-luminosity relations}

Color-luminosity (CL) relations for galaxies have been characterized by linear regressions of color (C) against absolute magnitude (M) (Baum 1959). Often C and M both include the same band, so that their errors are correlated. Most studies have also noted that the scatter about the linear CL regression is larger than can be explained by measurement error alone (e.g. Mobasher et al. 1986). Regressions for this type of data, then, fall exactly in the domain of the models developed in sections 2.1 and 3.

Almost without exception, studies of color-luminosity relations have used $\operatorname{OLS}\left(X_{2} \mid X_{1}\right)$, where $\mathrm{M}$ has been taken as the independent variable. These regressions typically do not weight by errors in $X_{2}(\mathrm{C})$, although several studies have included some form of robust 
estimation via iterative rejection of outlying points (Griersmith 1980, Bothun et al. 1985, Bower et al. 1992, and Bershady 1995). However, none of these studies have taken into account the correlation in the errors of color and magnitude. Wyse (1982) avoided this issue by fitting a linear regression directly to magnitudes in two bands.

To assess the magnitude of the biases present in analyses using incorrect statistical models, in Table 1 we compare a wide range of linear regression models fit to CL relations for two subsets of data from Bershady (1995). These subsets are defined as galaxies with spectral types $b k$, and type $a m$ or $f m$, where the type refers to the two stellar components dominating the galaxy's broad-band colors. "BCES" models include bivariate, correlated errors and intrinsic scatter (this paper). "BES" models include bivariate errors and intrinsic scatter, but without the correlated term $V_{12, i}$ (this paper). "OLS" models are those of IFAB, which include only homoscedastic intrinsic scatter. Finally, "WBE" models, for the $\left(X_{2} \mid X_{1}\right)$ case alone, include weighting by the bivariate errors, but no error correlation or intrinsic scatter (Bershady 1995). This method is formulated in terms of a minimizing $\chi^{2}$, as defined by Press et al. (1988), and solved numerically. [ Bershady's implementation of this method (used here) also includes iterative rejection of highly deviant points ( 5 standard deviations in error-normalized distance from the regression at each iteration). In several regards, therefore, this model is significantly different than any presented here. For each model the analytic estimates and standard deviations for $\beta$ and $\alpha$ are listed on the first line, with the results from 1000 simulations via bootstrap resampling on the following line.

As might be expected from the shallow slope and substantial scatter in the CL relation, the $\left(X_{1} \mid X_{2}\right)$ regressions (and therefore the bisectors) are steeper than the $\left(X_{2} \mid X_{1}\right)$ regressions. More subtle is the change (bias) with respect to models which include correlated errors and intrinsic scatter: slopes become steeper for $\left(X_{1} \mid X_{2}\right)$ and bisector regressions and shallower for $\left(X_{2} \mid X_{1}\right)$ and orthogonal regressions when correlated errors and intrinsic scatter are excluded from the regression models. For each family of models, orthogonal and $\left(X_{2} \mid X_{1}\right)$ regressions yield comparable slopes for these particular data sets. The $\operatorname{WBE}\left(X_{2} \mid X_{1}\right)$ model yields the shallowest slope.

What are the effects on possible scientific conclusions? If the CL relation is to be understood physically (e.g. Arimoto \& Yoshii 1987), then the "BCES" models should be used since they will give unbiased results. However, the variances for $\left(X_{2} \mid X_{1}\right)$ and orthogonal regressions are comparable for all models, with the exception that the boot-strap uncertainties are considerably smaller for $b k$ type galaxies for $\operatorname{WBE}\left(X_{2} \mid X_{1}\right)$. (In contrast

\footnotetext{
3 In Bershady (1995), this "WBE" method was attributed to be similar to one described by Stetson (1989); it is, however a substantially different statistical model.
} 
the variances for the $\left(X_{1} \mid X_{2}\right)$ and bisector regressions become larger when intrinsic scatter and measurement error are excluded from the statistical model.) As importantly, the regression slopes in these cases are the same for the two samples for a given model. One might therefore be tempted to conclude that the $\operatorname{OLS}\left(X_{2} \mid X_{1}\right)$ and WBE models adopted in previous studies are satisfactory for comparisons of CL regression slopes for different galaxy types (e.g. Mobasher et al. 1986 and Bershady 1995, respectively) or at different redshifts (Stanford et al. 1995). While this appears to be the case for the particular data set used here, in general OLS and WBE models yield biased results and therefore their estimated variances are not necessarily meaningful quantities since they do not include the effects of the unknown bias. Hence BCES models should be used even for slope comparisons between samples.

When using CL relations to estimate distance moduli (e.g. Sandage 1972), zeropoint differences between samples may be better estimated using cross-correlation techniques (e.g. Dressler 1984), as done by Bower et al. (1992).

Within the "BCES" family of models, which regression is best to use for CL-relation studies? To understand the bias and accuracy (due to small numbers) of each of these regressions, we conducted two simulation studies with artificially generated data sets designed to closely match the above observed color-luminosity distributions. One set of simulations, $\left(X_{1 i}, X_{2 i}\right), i=1, \ldots, n$, was generated according to the model in (3) with $\alpha_{1}=2.5$, and $\beta_{1}=0.07$. The range of the $X_{1}$-values was $(-28,-18)$ and the intrinsic scatter was generated according to a normal distribution with zero mean and standard deviation 0.55. Normal measurement errors were added to the $\left(X_{1}, X_{2}\right)$-values in order to simulate the observed data $\left(Y_{1 i}, Y_{2 i}\right), i=1, \ldots, n$. The range for the variances of the measurement errors was $(0.18,0.45)$, with the covariance fixed at 0.15 . A second set of simulations used $\beta_{1}=0.120 .12$, a measurement error on $X_{1}$ with variance ranging from $(0.03,0.3)$, a range of variance of the measurement error on $X_{2}$ of $(0.06,0.6)$, and a range of the covariance of the two measurement errors of $(0.03,0.3)$; all other parameters were the same as before. For both simulation sets, the randomly generated data $\left(Y_{1 i}, Y_{2 i}\right), V_{i}$ were fed into the BCES routine and the entire process was repeated 1000 times. The recorded outcome was the average (over the 1000 simulation runs) of the estimated coefficients, the sample variance of the the 1000 estimated coefficients, and the average value of the variance formulas for each of the estimated coefficients. Samples of $n=50,150$ and 500 were generated to understand the effects of small sample sizes on the estimated coefficients and variances.

For these simulation studies, $\hat{\beta}_{1}\left(\operatorname{BCES}\left(X_{2} \mid X_{1}\right)\right)$ performed best in all respects: Even with $n=50$ the bias (small-sample bias) was small and the sample variance over the 1000 simulations closely matched the average variance computed from the formula. The 
variance of $\hat{\beta}_{1}$ was the smallest of all the estimators (a factor of 4 better than the next smallest variance). There was no noticeable change in the performance of $\operatorname{BCES}\left(X_{2} \mid X_{1}\right)$ for the different sample sizes. The performance of the other estimators did change with the sample size. When the true $\beta_{1}$ slope was $0.07, \operatorname{BCES}\left(X_{1} \mid X_{2}\right)$ and BCES-orthogonal

regressions had considerable biases in their slopes $\hat{\beta}_{2}$ and $\hat{\beta}_{4}$ for $n=50$. When $\beta_{1}$ was set to 0.12 , BCES-orthogonal regression slope, $\hat{\beta}_{4}$, performed better than $\operatorname{BCES}\left(X_{1} \mid X_{2}\right)$ and BCES-bisector regressions slopes $\hat{\beta}_{2}$ and $\hat{\beta}_{3}$ in terms of both bias and variance for $n=150$ and 500. On the basis of these simulation results we recommend the use of $\operatorname{BCES}\left(X_{2} \mid X_{1}\right)$ for color-luminosity data sets similar to those presented here. An important caveat is that different model specifications might result in different performance of the estimators. This should be checked for each specific study.

\subsubsection{The Tully-Fisher relation}

Another example of data with correlated errors is the relation between spectral line-width (internal velocity) and luminosity of spiral galaxies (Tully \& Fisher 1977). Here too there exists a dispersion about the linear regression in addition to measurement error (e.g. Pierce \& Tully 1992). The error correlation occurs because both the velocity (corrected for projection) and absolute magnitude (corrected for dust extinction) depend on the inferred inclination. There can be non-negligible uncertainties in the inclination measurement, particularly for galaxies that are not spatially well-resolved. The effect of the correlated magnitude and velocity errors will be to increase the observed scatter and hence make the observed relation too flat if unaccounted for. In all cases, linear regressions should be computed for the Tully-Fisher relation using the BCES model in preference over other existing models.

We note however, that the BCES model is not immune to biases that arise from data truncation (i.e. a luminosity-limited sample, or a magnitude-limited sample in the case of a cluster). This has been explored in detail by Willick (1994, and references therein) for the case of intrinsic scatter but uncorrelated errors. Another limitation of the BCES model is that it does not allow for changes in the scatter along the regression. The sample of Mathewson et al. (1992) suggests that the scatter in the current Tully-Fisher relation (as defined by 21-cm integrated line-widths) increases at lower velocities or luminosities. Future work should consider elaborations of the BCES statistical model to include variable intrinsic scatter, as well as estimation of this scatter. 


\subsection{Errors in $X_{2}$ only and intrinsic scatter: The Tolman Test}

A fundamental test in observational cosmology is verification that redshift is caused by a secular change in the metric (Tolman 1930), namely universal expansion. If true, then surface-brightness in a fixed band-pass scales as the kinematic factor $(1+z)^{-3}$, independent of other cosmological parameters (although for astrophysical sources such as galaxies, the dimming is modified by the $K$-correction). There have been several recent attempts to perform the Tolman test (Sandage \& Perelmuter 1990, Kjaergaard, Jorgensen \& Moles 1993, and Pahre, Djorgovski \& de Carvalho 1996). These studies note that the rest-frame surface-brightnesses of galaxies are not all the same, but depend on a number of variables, including luminosity or size. However, even after taking this into account, galaxy samples are likely to still have some intrinsic dispersion in surface-brightness at a given redshift. However, in terms of measurement errors, redshifts can typically be measured with high precision compared to the apparent magnitudes and sizes needed to derive surface-brightnesses. Hence, the linear regression for surface-brightness vs. $\log (1+z)$ for such a data set is well approximated by the WLS model presented here.f In fact, since the WLS model includes (and estimates) intrinsic scatter, it is not necessary to "reduce" the data to some fiducial surface-brightness on the basis of luminosity or size. This has the distinct advantage of allowing the test to be extended to galaxies for which tight "Fundamental Plane" relations have not yet been formulated.

We have tested the WLS method with two simulation studies. The first simulated data sets were generated as described in the simulations reported in subsection 4.2.1, but without adding the error in the $X_{1}$ variable. The small-sample bias of the WLS estimator was comparable to that of $\operatorname{BCES}\left(X_{2} \mid X_{1}\right)$, but the variance of the WLS estimator was an order of magnitude smaller than that of $\operatorname{BCES}\left(X_{2} \mid X_{1}\right)$ ! The same results were found for the second simulated data set with parameters designed to mimic the Tolman test in the $K$ band, assuming surface-brightnesses are measured in large, metric apertures to redshifts of $\sim 0.4$, and $K$-corrected surface-brightnesses are plotted versus $2.5 \log (1+\mathrm{z}): \alpha_{1}=16, \beta_{1}$ $=4, X_{1}$ in the range $(0,0.4)$, intrinsic scatter given by a normal distribution with zero mean and standard deviation of 0.3 , and normal measurement errors on $X_{2}$ with variances in the range $(0.03,0.3)$. However, the formula for the confidence interval on $\hat{\beta}_{1}$ for this second study gave conservative results (i.e. wider confidence intervals) even for sample sizes of 500. Only for sample sizes of 900 did the formula for the confidence interval capture the true variability of the WLS estimator. This may be due to the narrow range of the

\footnotetext{
${ }^{4}$ Any linear correlation as a function of redshift is likely to fall in this category for astrophysical sources.
} 
$X_{1}$-values (the confidence interval formula performed well for much smaller numbers for the first simulation set).

On the basis of these simulation results we recommend the use of the WLS estimator whenever the $X_{1}$ variable is observed without measurement error and the magnitudes of the measurement errors can be assumed independent from the observations. This would be

case for the Tolman test, for example, when flux measurements are background-limited. As an additional bonus, the WLS provides an estimate of the intrinsic scatter. However, for a narrow range of $X_{1}$-values, we recommend the use of bootstrap confidence intervals even for relatively large sample sizes.

\section{Discussion}

To our knowledge, the methods presented here are the only algorithms that apply to data with both measurement errors and intrinsic scatter. When is it necessary to use one of the above methods over the techniques discussed in IFAB or FB? There are two basic criteria for selecting a statistical model to use for studying correlations in data, bias and uncertainty. Their relative importance depends somewhat on the specific scientific objective, however the conclusion is the same: When in doubt, the BCES and WLS models should be used. However, the WLS model should be used only in the approximation where the $X_{1}$ variable is measured without error.

If the purpose is to test a theory which predicts correlation slopes and/or zeropoints for some set of observables, then bias is the principal criterion. The statistical model which best approximates the real data is expected to give the least-biased regression, and so the choice becomes an issue of approximation. Because astronomy largely consists of passive observations and not active experiments, there is rarely an 'explanatory' variable free of measurement error. Moreover, correlations between variables for astronomical systems almost always have intrinsic scatter, which is simply a reflection of these systems' complex, multi-variate dependencies. The 'Fundamental Plane' for elliptical galaxies is one good astronomical example of this complexity (cf. Santiago \& Djorgovski 1993). For cases where the intrinsic scatter may be much larger than measurement error, or vice-versa, the methods in IFAB or those outlined in FB, respectively, may provide acceptable approximations. However, at this time we cannot quantify "much larger". The methods presented here are valid in general and, since they reduce to the methods considered in IFAB in the case of no measurement errors, we recommend that the present methods be used in all cases. 
There are some situations where differential measurements are designed simply to detect differences in slope between samples. Examples of this were described for the CL relation. Here, the most accurate regression estimate may be desired, and should be assessed via simulations of artificial data sets, as we have illustrated for the BCES family of models. However, if the statistical model is incorrect, then the estimated variance does not necessarily include effects of bias, which may differ from sample to sample. While bootstrap estimates of the variance may be 'unbiased,' the same is not necessarily true of the slope. To put it another way, if the null hypothesis is that two samples are the same, and this is to be confirmed by comparing regression properties, any statistical model may yield results consistent with the hypothesis. However, if the statistical model is incomplete or unrepresentative of the data, the comparison is only consistent and cannot validate the hypothesis. Again, BCES models are the most general and should provide the least-biased estimates of regression slopes and variances.

Within a family of regressions models (e.g. BCES or OLS), the choice of particular regression $\left(\left(X_{2} \mid X_{1}\right),\left(X_{1} \mid X_{2}\right)\right.$, etc. $)$ is only an issue of accuracy, and not bias. As has been emphasized in IFAB and again here, the different regression methods give different slopes even at the population level. All slopes are related to the second moments of the bivariate distribution of the data. Again, the most accurate regression should be assessed via simulations.

In the case where the $X_{1}$ variable is measured without error, our simulations for two different artificial data sets revealed that the WLS estimator has smaller variance than $\operatorname{BCES}\left(X_{2} \mid X_{1}\right)$. However WLS is consistent only when the error magnitude is independent from the observation. While the BCES estimators are consistent under general conditions, the simulations suggest they can be improved under the additional assumption that the measurement errors on $X_{1}, X_{2}$ are independent from the observations. Weighted versions of the BCES estimators under this additional assumption will be the subject of a forthcoming paper.

The present procedures resulted from an interdisciplinary collaboration of astrophysicists and mathematical statisticians via the newly founded Statistical Consulting Center for Astronomy (SCCA). Further information about SCCA can be obtained through the World Wide Web (http://www.stat.psu.edu/scca/homepage.html), or by contacting SCCA@stat.psu.edu. A FORTRAN package which includes the algorithms in this paper and IFAB, including bootstrap resampling error analysis, is available via anonymous ftp (contact mab@astro.psu.edu).

The work of MGA was supported in part by NSF grant DMS-9208066. MAB acknowledges support from NASA through grant HF-1028.02-92, from the Space Telescope 
Science Institute, which is operated by the Association of Universities for Research in Astronomy, Incorporated, under contract NAS5-26555.

\section{A. Proofs}

Proof of Relations (5), (6) and (7). Relation (5) is obvious from relation (2) and the fact that, conditionally on $V_{k k, i}$, the errors $\epsilon_{k i}, k=1,2$ have zero mean. To show (6), note that

$$
\begin{aligned}
E\left(Y_{k i}^{2}\right) & =E\left[E\left(Y_{k i}^{2} \mid V_{k k, i}\right)\right] \\
& =E\left[E\left(\left(Y_{k i}-X_{k i}\right)^{2}+X_{k i}^{2}+2 X_{k i}\left(Y_{k i}-X_{k i}\right) \mid V_{k k, i}\right)\right] \\
& =E\left[E\left(\epsilon_{k i}^{2}+X_{k i}^{2}+2 X_{k i} \epsilon_{k i} \mid V_{k k, i}\right)\right] \\
& =E\left(V_{k k, i}\right)+E\left(X_{k i}^{2}\right) .
\end{aligned}
$$

Since the variance of any random variable $Z$ is $V(Z)=E\left(Z^{2}\right)-[E(Z)]^{2}$, (6) follows from the above relation and (5). Similarly, the proof of (7) follows from

$$
\begin{aligned}
E\left(Y_{1 i} Y_{2 i}\right) & =E\left[E\left(Y_{1 i} Y_{2 i} \mid \mathbf{V}_{k i}\right)\right] \\
& =E\left[E\left(\epsilon_{1 i} \epsilon_{2 i}+X_{1 i} X_{2 i}+X_{1 i} \epsilon_{2 i}+X_{2 i} \epsilon_{1 i} \mid \mathbf{V}_{k i}\right)\right] \\
& =E\left(V_{12, i}\right)+E\left(X_{1 i} X_{2 i}\right)
\end{aligned}
$$

the fact that the covariance of any two random variables $Z_{1}, Z_{2}$, is $\operatorname{Cov}\left(Z_{1}, Z_{2}\right)=$ $E\left(Z_{1} Z_{2}\right)-E\left(Z_{1}\right) E\left(Z_{2}\right)$ and from (5).

Proof of Asymptotic Normality of $\hat{\beta}_{1}$. Write $S_{Y_{1}, Y_{2}}=n^{-1} \sum_{i=1}^{n}\left(Y_{1 i}-\bar{Y}_{1}\right)\left(Y_{2 i}-\bar{Y}_{2}\right)$, and $S_{Y_{1}}^{2}=n^{-1} \sum_{i=1}^{n}\left(Y_{1 i}-\bar{Y}_{1}\right)^{2}$. We will need the following relations.

$$
\begin{aligned}
\sqrt{n}\left(S_{Y_{1}, Y_{2}}-C\left(Y_{1}, Y_{2}\right)\right) & =n^{-1 / 2} \sum_{i=1}^{n}\left(Y_{1 i} Y_{2 i}-E\left(Y_{1} Y_{2}\right)\right)-E\left(Y_{1}\right) n^{1 / 2}\left(\bar{Y}_{2}-E\left(Y_{2}\right)\right) \\
& -E\left(Y_{2}\right) n^{1 / 2}\left(\bar{Y}_{1}-E\left(Y_{1}\right)\right)+o_{p}(1) \\
\sqrt{n}\left(S_{Y_{1}}^{2}-V\left(Y_{1}\right)\right) & =n^{-1 / 2} \sum_{i=1}^{n}\left(Y_{1 i}^{2}-E\left(Y_{1}^{2}\right)\right)-2 E\left(Y_{1}\right) n^{1 / 2}\left(\bar{Y}_{1}-E\left(Y_{1}\right)\right) \\
& +o_{p}(1)
\end{aligned}
$$


where $o_{p}(1)$ denotes a quantity that converges to zero in probability as $n \rightarrow \infty$. Write

$$
\begin{aligned}
\sqrt{n}\left(\hat{\beta}_{1}-\beta_{1}\right) & =\sqrt{n}\left[\frac{S_{Y_{1}, Y_{2}}-\bar{V}_{12}}{S_{Y_{1}}^{2}-\bar{V}_{11}}-\frac{C\left(Y_{1 i}, Y_{2 i}\right)-E\left(V_{12, i}\right)}{V\left(Y_{1 i}\right)-E\left(V_{11, i}\right)}\right] \\
& =\sqrt{n}\left[\frac{S_{Y_{1}, Y_{2}}-C\left(Y_{1 i}, Y_{2 i}\right)-\left(\bar{V}_{12}-E\left(V_{12, i}\right)\right)}{V\left(Y_{1 i}\right)-E\left(V_{11, i}\right)}\right. \\
& \left.-\left[C\left(Y_{1 i}, Y_{2 i}\right)-E\left(V_{12, i}\right)\right] \frac{S_{Y_{1}}^{2}-V\left(Y_{1 i}\right)-\left(\bar{V}_{11}-E\left(V_{11, i}\right)\right.}{\left[V\left(Y_{1 i}\right)-E\left(V_{11, i}\right)\right]^{2}}\right]+o_{p}(1)
\end{aligned}
$$

Using (A1) and (A2), it can be seen after some algebra that (A3) can be written as

$$
\sqrt{n}\left(\hat{\beta}_{1}-\beta_{1}\right)=\sqrt{n}\left(\bar{\xi}_{1}-E\left(\xi_{1 i}\right)\right)+o_{p}(1)
$$

and an application of the Central Limit Theorem completes the proof of the asymptotic normality. That (13) and (14) provide consistent estimators of $\sigma_{\beta_{1}}^{2}$ and $\sigma_{\alpha_{1}}^{2}$ is straightforward.

Acknowledgment. Thanks are due to J. Willick for many constructive comments that greatly improved the presentation of the paper. 


\section{REFERENCES}

Arimoto, N., \& Yoshii, Y. 1987, A\&AS, 173, 23

Arnold, S.F. 1981, The Theory of Linear Models and Multivariate Analysis, (New York: Wiley), p. 204

Baum, W.A. 1959, PASP, 71, 106

Bershady, M.A. 1995, AJ, 109, 87

Boggs, P.T., Donaldson, J.R., Byrd, R.H. \& Schnabel, R.B. 1990, ACM Trans. Math. Software, 15, 348

Bothun, G.D., Mould, J., Schommer, R.A. \& Aaronson, M. 1985, ApJ, 291, 586

Bower, R.G., Lucey, J.R. \& Ellis, R.S. 1992, MNRAS, 254, 601

Draper, N.R. \& Smith, H. 1981, Applied Regression Analysis, (New York: Wiley), p. 24, 27

Dressler, A. 1984, ApJ, 281, 512

Feigelson, E.D. \& Babu, G.J. 1992, ApJ, 397, 55

Fuller, W.A. 1987, Measurement Error Models, (New York: Wiley)

Isobe, T., Feigelson, E.D., Akritas, M.G. \& Babu, G.J. 1990, ApJ, 364, 104

Johnson, R.A. \& Wichern, D.W. 1988, Applied Multivariate Statistical Analysis, (Englewood Cliffs: Prentice Hall), p. 180

Kjaergaard, P., Jorgensen, I., Moles, M. 1993, ApJ, 418, 617

Mathewson, D.S., Ford, V.L. \& Buchhorn, M. 1992, ApJS, 81, 413

Mobasher, B., Ellis, R.S. \& Sharples, R. M. 1986, MNRAS, 223, 11

Pahre, M.A., Djorgovski, S.G., de Carvalho, R.R. 1996, 456, L79

Pierce, M.J. \& Tully, R.B. 1992, ApJ, 387, 47

Press, W. H., Teukolsky, S. A., Vetterling, W. T., Flannery, B. P. 1988, "Numerical Recipes in C," (Cambridge University Press), p. 666

Sandage, A. 1972, ApJ, 173, 485

Sandage, A., \& Perelmuter, J.-M. 1991, 370, 455

Santiago, B.X. \& Djorgovski, S. 1993, MNRAS, 261, 753

Stanford, S.A., Eisenhardt, P.R.M. \& Dickinson, M. 1995, ApJ, in press 
Stetson, P.B. 1989, The Techniques of Least Squares and Stellar Photometry With CCDs, a series of five lectures presented at V Escola Avançada de Astrofisca (Dominion Astrophysical Observatory, Victoria)

Tolman, R.C., 1930, Proc. Nat. Acad. Sci., 16, 511

Tully, R.B. \& Fisher, J.R. 1977, å, 54, 661

Willick, J.A. 1991, Ph.D. thesis, University of California, Berkeley

Willick, J.A. 1994, ApJS, 92, 1

Wyse, R. 1982, MNRAS, 199, 1P 
TABLE 1

Regressions For The Color-luminosity Relation: $\left(V^{\prime}-K\right)_{0}=\beta M_{K}+\alpha$

\begin{tabular}{|c|c|c|c|c|c|c|c|c|}
\hline \multirow[b]{2}{*}{ fit } & \multicolumn{4}{|c|}{$b k$ type galaxies $(\mathrm{N}=34)$} & \multicolumn{4}{|c|}{$a m, f m$ type galaxies $(N=60)$} \\
\hline & $\hat{\beta}$ & $\hat{\sigma}(\beta)$ & $\hat{\alpha}$ & $\hat{\sigma}(\alpha)$ & $\hat{\beta}$ & $\hat{\sigma}(\beta)$ & $\hat{\alpha}$ & $\hat{\sigma}(\alpha)$ \\
\hline \multirow{2}{*}{$\operatorname{BCES}\left(X_{2} \mid X_{1}\right)$} & -0.123 & 0.034 & -0.22 & 0.79 & -0.114 & 0.018 & 0.48 & 0.46 \\
\hline & -0.126 & 0.045 & -0.28 & 1.04 & -0.113 & 0.022 & 0.52 & 0.55 \\
\hline \multirow[t]{2}{*}{$\operatorname{BCES}\left(X_{1} \mid X_{2}\right)$} & -0.179 & 0.053 & -1.46 & 1.21 & -0.273 & 0.107 & -3.46 & 0.26 \\
\hline & -0.196 & 0.035 & -1.83 & 0.81 & -0.328 & 0.153 & -4.82 & 0.38 \\
\hline \multirow[t]{2}{*}{ BCES Bisector } & -0.151 & 0.039 & -0.84 & 0.91 & -0.193 & 0.050 & -1.46 & 0.12 \\
\hline & -0.160 & 0.034 & -1.05 & 0.79 & -0.216 & 0.060 & -2.03 & 0.15 \\
\hline \multirow[t]{2}{*}{ BCES Orthogonal } & -0.124 & 0.034 & -0.24 & 0.72 & -0.116 & 0.018 & 0.43 & 0.45 \\
\hline & -0.127 & 0.044 & -0.30 & 1.03 & -0.115 & 0.022 & 0.46 & 0.54 \\
\hline \multirow[t]{2}{*}{$\operatorname{BES}\left(X_{2} \mid X_{1}\right)$} & -0.106 & 0.032 & 0.16 & 0.75 & -0.097 & 0.019 & 0.90 & 0.48 \\
\hline & -0.108 & 0.034 & 0.12 & 0.80 & -0.094 & 0.021 & 0.97 & 0.52 \\
\hline \multirow[t]{2}{*}{$\operatorname{BES}\left(X_{1} \mid X_{2}\right)$} & -0.208 & 0.050 & -2.09 & 1.14 & -0.321 & 0.118 & -4.63 & 2.92 \\
\hline & -0.229 & 0.052 & -2.57 & 1.19 & -0.399 & 0.244 & -6.58 & 6.05 \\
\hline \multirow[t]{2}{*}{ BES Bisector } & -0.157 & 0.036 & -0.96 & 0.84 & -0.207 & 0.052 & -1.81 & 1.29 \\
\hline & -0.167 & 0.036 & -1.21 & 0.84 & -0.236 & 0.073 & -2.53 & 1.81 \\
\hline \multirow[t]{2}{*}{ BES Orthogonal } & -0.108 & 0.033 & 0.13 & 0.69 & -0.100 & 0.019 & 0.84 & 0.47 \\
\hline & -0.109 & 0.035 & 0.09 & 0.81 & -0.097 & 0.021 & 0.91 & 0.53 \\
\hline \multirow[t]{2}{*}{$\operatorname{OLS}\left(X_{2} \mid X_{1}\right)$} & -0.105 & 0.031 & 0.20 & 0.72 & -0.096 & 0.019 & 0.94 & 0.48 \\
\hline & -0.108 & 0.034 & 0.12 & 0.79 & -0.094 & 0.021 & 0.98 & 0.52 \\
\hline \multirow[t]{2}{*}{$\operatorname{OLS}\left(X_{1} \mid X_{2}\right)$} & -0.342 & 0.124 & 5.09 & 2.78 & -0.450 & 0.139 & -7.83 & 3.46 \\
\hline & -0.329 & 0.121 & -4.79 & 2.73 & -0.521 & 0.286 & -9.60 & 7.10 \\
\hline \multirow[t]{2}{*}{ OLS Bisector } & -0.220 & 0.067 & -2.38 & 1.54 & -0.265 & 0.056 & -3.25 & 1.39 \\
\hline & -0.215 & 0.067 & -2.26 & 1.54 & -0.287 & 0.077 & -3.80 & 1.91 \\
\hline \multirow[t]{2}{*}{ OLS Orthogonal } & -0.107 & 0.032 & 0.14 & 0.69 & -0.099 & 0.019 & 0.85 & 0.47 \\
\hline & -0.111 & 0.036 & 0.05 & 0.85 & -0.098 & 0.021 & 0.89 & 0.53 \\
\hline \multirow[t]{2}{*}{ WBE $\left(X_{2} \mid X_{1}\right)$} & -0.083 & $\ldots$ & 0.68 & $\ldots$ & -0.095 & $\ldots$ & 0.96 & $\ldots$ \\
\hline & -0.083 & 0.019 & 0.69 & 0.43 & -0.094 & 0.023 & 0.97 & 0.59 \\
\hline
\end{tabular}

